\title{
HACIA UNA TEORÍA SOBRE LAS IDEAS CIENTÍFICAS DE LOS ALUMNOS: INFLUENCIA DEL CONTEXTO
}

\author{
DE POSADA, J.M. \\ Delegación provincial de Educación y Ciencia de Málaga. Junta de Andalucía.
}

\section{SUMMARY}

The different constructive psychological theories present some difficulties in order to explain and justify the results obtained by the researches carried out within the field named pupils'conceptions. In this paper we introduce a new psycho-pedagogical model which agglutinates the main elements of the constructive theories. With that aim, the declarative knowledge is subdivided in several memories: episodic, scholastic semantic and experiential semantic. The procedural knowledge of reasoning in scholastic analysis and experiential analysis methods. We show some examples that are analyzed from our theoretic model. Later, we stress the relationship between pupils' answers and the context in which those are activated, showing the utility of the model here proposed.

\section{INTRODUCCIÓN}

Se han identificado nociones de mecánica, termología, evolución biológica, presión de gases, electricidad, energía, luz, reacciones químicas, conservación de la masa, equilibrio químico, radioactividad, etc. en las mentes de los alumnos incluso antes de haber sido impartidos formalmente en el currículo. En otros casos, y después de haber sido tratados determinados conceptos en clase, se detectan ideas de naturaleza distinta a la que se esperaba. La lista de nociones identificadas continúa creciendo sin cesar, así como el volumen de datos sobre las mismas. EI interés no parece disminuir a juzgar por el gran número de comunicaciones orales presentadas sobre el tema en el IV Congreso Internacional sobre investigación en la Didáctica de las Ciencias y de las Matemáticas celebrado en septiembre de 1993 en Barcelona. Hemos computado 63 trabajos de un total de 167 presentados, lo que supone un $38 \%$ del total.

A pesar de las grandes diferencias encontradas entre los topicos estudiados, Driver, Guesne y Tiberghien (1985) reconocieron algunas características comunes en las representaciones de los estudiantes:
Inicialmente los alumnos tienden a basar su razonamiento en características observables de la situación problema. La tierra es plana para muchos estudiantes y el azúcar «desaparece» al ser disuelto en agua.

Los estudiantes consideran aspectos muy limitados y focalizan su atención sobre las características más sobresalientes.

Los alumnos tienden a fijarse más en los cambios que en las situaciones estables. El razonamiento utilizado para explicar los cambios parece seguir una secuencia causal Inneal.

Algunas de las representaciones de los estudiantes tienen un rango de aplicación que difieren del de los científicos. Por ejemplo, la noción de peso tiene connotaciones de volumen, densidad y presión para los estudiantes más jóvenes.

Existe un determinado número de representaciones, mencionadas frecuentemente en la literatura, que influ- 
yen en el pensamiento de los estudiantes en situaciones muy variadas.

Disponemos pues de un valioso catálogo de cómo los alumnos entienden muchos conceptos científicos y de evidencias útiles acerca de la resistencia al cambio de los errores conceptuales de los alumnos. Sin embargo, no tenemos en la actualidad un esquema térico que pueda explicár estos hallazgos (Lawson, 1989; Marín y Benarroch, 1994; Niaz, 1993): es necesario desarrollar un modelo teórico que pueda ser refutado (Adey, I987). La teoría del aprendizaje significativo de Ausubel et al. (1978) destaca la importancia de las ideas previas de los estudiantes; sin embargo, no recibe un tratamiento adecuado en la misma la persistencia y naturaleza de estas concepciones, que son fundamentales en determinados niveles de escolaridad y en algunos núcleos conceptuales como mecánica, calor, óptica, etc. (Gómez et al., 1993a). Las ideas de Vygotski (1962) sobre las relaciones entre conceptos espontáneos y científicos son oportunas; sin embargo, deja sin contestar algunas cuestiones como la falta de criterios suficientes para determinar cuándo un concepto pertenece a un sistema o a otro (Davydov, 1972; en Pozo, 1989). La teoría causal de Piaget (1974) tampoco se encuentra exenta de críticas. Para algunos investigadores se debería focalizar más en el contenido real de las ideas de los alumnos y menos en su supuesta estructura lógica (Posner et al., 1982). Para Lacasa y García Madruga (1987) y Gilbert y Swift (1985), el modelo piagetiano es ante todo epistemológico y, por tanto, no está formulado para dar cuenta de una práctica experimental concreta. De acuerdo con Vuyk (1985), la teoría de Piaget tiene un carácter descriptivo pero no explicativo. Aunque en la teoría del procesamiento de la información existen actualmente tendencias de aproximación hacia posiciones constructivistas (Streibel, 1989), su núcleo central se encuentra aún comprometido con presupuestos conductistas (Sebastián, 1983; Pozo, 1989).

Estos comentarios previos no pretenden ser un catălogo de las principales críticas encontradas en la bibliografía. Por el contrario, se trata de breves pinceladas que ponen de manifiesto las dificultades para interpretar de forma teórica los resultados obtenidos sobre los esquemas de los alumnos. Efectivamente, numerosos investigadores (Corral, 1986; Lacasa y García Madruga, 1987; Coll, $199 \mathrm{la}$; entre otros) coinciden en destacar la falta de un marco teórico general para la educación, pues ni siquiera existe para el campo de investigación más restringido como es el de los esquemas de los alumnos (Posner et al., 1982; Pozo, 1989; Giordan y de Vecchi, 1988, entre otros). Este hecho podría explicar la dispersión teórica, metodológica, uso de nomenclaturas, etc. encontradas en esta área (Marín y Jiménez, 1992); así, por ejemplo, se utilizan hasta 28 términos diferentes para referirse a los constructos de los estudiantes (Giordan y de Vecchi, 1988). Estamos totalmente de acuerdo con Porlán (1993) cuando afirma que es necesaria una profundización y unificación integradora de los fundamentos psicológicos para la didáctica de las ciencias.

Los objetivos de este artículo son:
Reclamar una mayor atención sobre la necesidad de unificar criterios y esfuerzos para conseguir un marco teórico más general para las concepciones de los alumnos.

Presentar un nuevo modelo psicopedagógico del que se puede deducir los aspectos más destacados y consensuales de las principales corrientes constructivistas.

Explicar algunas características de las concepciones alternativas de los alumnos desde el modelo integrador.

Establecer relaciones entre los contextos en los que son planteadas tareas y los elementos de memoria que son puestos en juego en la respuesta.

\section{UN MARCO INTEGRADOR}

Ryle (1949) realizó una útil diferenciación del conocimiento en dos tipos: declarativo y procedural. El conocimiento declarativo comprende el saber descriptivo o factual susceptible de ser expresado. El conocimiento procedural se describe como todos aquellos procedimientos disponibles por el individuo para actuar sobre su entorno. Esta diferenciación sigue siendo utilizađa tanto en modelos psicológicos como los de Anderson (1980, 1983) y Gagné y otros (1988) como en el modelo epistemológico de Piaget (1971); en este ứtimo con las denominaciones de figurativo y operativo para referirse respectivamente, según Lawson (1982), a declarativo y procedural'. Esta diferenciación del conocimiento cuenta con suficiente respaldo neurológico. La zona posterior del cerebro humano se encarga de la recepción y almacenaje del conocimiento declarativo (Cardebat et al., 1994), mientras que la porción frontal dei cerebro parece ser la responsable de la organización y generación de acciones del conocimiento procedural (Anderson, 1975; Luria, 1973; Levine y Prueitt, 1989, entre otros).

Tulving (1972) introdujo la distinción entre memoria semántica y memoria episódica. Para Tulving y Thomson (1973), la memoria episódica no tiene una organización conceptual aunque sí espacio-temporal; esta memoria está relacionada con los recuerdos personales. La memoria semántica almacena conocimientos permanentes sin relación con momentos concretos en los que fueron adquiridos dichos conocimientos. La memoria semántica tiene una estructura organizada jerárquicamente (Collins y Quillian, 1969) que podría no estar asociada a la estructura lógica (Rips et aI., 1973). Este modelo está demostrando su utilidad en los problemas humanos de almacenaje y recuperación de clementos de Ia memoria (Baddeley, 1994). Las memorias semántica y experiencial, por su naturaleza, pueden ser fácilmente incluidas dentro deI conocimiento declarativo. Por otro lado, Ausubel y otros (1978) consideran que el aprendizaje, escolar o no, puede ser analizado de acuerdo con un continuo que va del aprendizaje rutinario al significativo. Éste se produce cuando el nuevo conocimiento «puede relacionarse, de modo no arbitrario y substancial (no al pie de la letra) con lo que el alumno ya sabe" 
(Ausubel et al., 1978, pág. 37 de la trad. cast.). Los términos aprendizaje y memoria son usados por algunas teorías de forma casi sinónima y, sin embargo, son dos realidades diferentes (Calfee, 1981; Bereister, 1990; Baddeley, 1994) que conviene distinguir en el nuevo modelo que presentamos.

Una propuesta útil para resolver algunos de los problemas teóricos y metodológicos expuestos anteriormente es la organización y diferenciación de la memoria declarativa en tres niveles: memoria episódica, memoria semántica academicista y memoria semántica experiencial (Pérez Gómez, 1988, Fig. 1). La memoria episódica comprende aqueltos recuerdos, experiencias, etc. sin conexión unos con otros, producto de la práctica diaria directamente perceptiva de la persona. La memoria semántica academicista alberga el conjunto de concepciones que fueron adquiridas mediante un aprendizaje memorístico, no sustantivo, en los que no se integran esos nuevos conceptos con los que existen previamente en la estructura cognitiva. Esas concepciones no son relacionadas por el alumno con experiencias, hechos u objetos conocidos por ćl. Numerosos investigadores (Ridley y Novak, 1983; Novak, 1988; Tobin y Fraser, 1989) han mostrado que muchos estudiantes aprenden básicamente de forma rutinaria. El término academicista no implica connotación negativa hacia lo aprendido en el ámbito escolar. La memoria semántica experiencial acoge las concepciones incorporadas substantivamente, de forma no arbitraria y sí jerárquicamente ${ }^{2}$, que se encuentran relacionadas con hechos, experiencias u objetos conocidos por el alumno ${ }^{3}$. Las concepciones ubicadas en la memoria semántica academicista pueden pasar a ja semántica experiencial si el individuo consigue relacionarlas significativamente con las que ya posee.

Para aclarar estos términos usaremos la experiencia descrita por White (1988) en su libro Learning Science (pp. 31 -32). Este investigador recuerda que cuando tenía quince años tuvo una conversación con su primo y aún la rememora con todo detalle. Su primo le informó que podía haber aceleración sin cambiar de velocidad. En la discusión que tuvo lugar, las nuevas ideas aportadas por su primo se encontraron relacionadas con conceptos previamente bien conocidos por cl joven White como eran, rapidez, aceleración, vector velocidad, etc. (todos ellos ubicados en su memoria semántica experiencial)

Figura 1

Tipos de memoria declarativa.

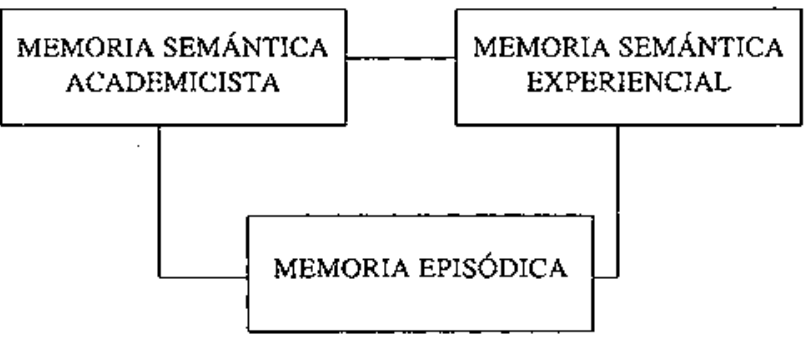

aunque posiblemente nunca antes había visto el problema desde la optica planteada hasta ese momento. En las clases tradicionales de física se suele definir el vector aceleración como la derivada del vector velocidad en función del tiempo. El tipo de información así presentada suele situarse en la memoria semántica academicista de la mayoría de los estudiantes de esta edad y no tiene las cualidades de la memoria semántica experiencial, como veremos posteriormente. El hecho de encontrarse con stl primo en un tren, recordar la ropa que llevaban puesta, etc. debe ser considerado episódico, sin embargo, el conocimiento construido en la mente de White se ubicó en su memoria semántica experiencial y perdura hasta hoy.

Al esquema de la figura 1 han sido agregados varios apartados para dar cuenta de algunos hechos que hemos podido observar (de Posada, 1993a, Fig. 2). El cuerpo de conocimientos científicos imperantes, lógicamente, es mayor que el que posee un individuo determinado, habida cuenta la velocidad a la que se desarrollan nuevos campos de la ciencia y la imposibilidad material de estar al tanto de todos los nuevos avances. Dentro de la memoria semántica academicista existen conocimientos que están de acuerdo con lo establecido por la comunidad científica (conjunto de conocimiento representado por la zona 1 de la Fig. 2). Muchos estudiantes de grado medio recuerdan las leyes de Mendel, los principios de la mecánica o el principio de conservación de la masa; sin embargo no llegan a captar su significado. Si se les plantean tareas en las que deben utilizar los

Figura 2

Diagrama que representa la memoria declarativa y el papel que juegan los factores inclusores en la producción de aprendizaje significativo. Las aclaraciones I a 4 son explicadas en el texto.

\section{MEDIO FSCOLAR Y EXTRAESCOLAR} $\downarrow \quad \downarrow \quad \downarrow$

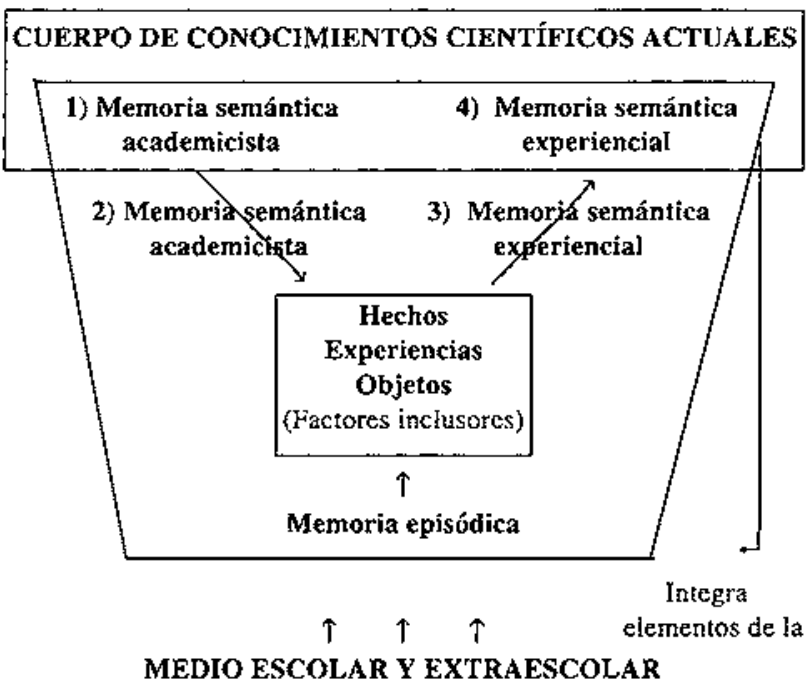


conceptos científicos, cuyos principios y leyes recuerdan perfectamente, estos alumnos suelen utilizar otras concepciones que no son las científicas. Hay otros conocimientos en la memoria semántica academicista que son producto de malas interpretaciones, enseñanzas o aprendizajes defectuosos que no están de acuerdo con los prescritos por la ciencia (zona 2 de la Fig. 2). A veces surgen como consecuencia đe la simplificación excesiva de conceptos e ideas, como en la respuesta siguiente dada por un alumno: «En agua se disuelve lo inorgánico y en el tetracloruro de carbono los compuestos orgáni$\cos . \gg\left(3^{\circ}\right.$ de BUP, 17 años de edad). Respuestas de este tipo son bastante frecuentes; todas tienen en común una falta real de comprensión de lo que se dice o escribe por parte del alumno.

Las conexiones entre conceptos o los propios conceptos de la memoria semántica experiencial pueden estar en desacuerdo con la ciencia (zona 3, Fig. 2) o de acuerdo con ella. Este último es el objetivo final de la enseñanza científica. Los individuos acumulamos experiencias, hechos, recuerdos, etc. y algunos conectan con nuestras concepciones previas (son asimilados o interiorizados) mientras que otros no lo consiguen. El conocimiento previo de los estudiantes ha sido reconocido como el factor conceptual más importante para comprender la adquisición de nuevos conocimientos (Ausubel et al., 1978; Calfee, 1981; Corno, 1981; Posner et al., 1982; Johnson-Laird, 1983; Osborne y Wittrock, 1983, 1985; Alexander et aI., 1991; Nuthall y Alton-Lee, 1993, entre otros). Las experiencias, hechos, recuerdos, etc. que conectan con los conocimientos previos asumen una posición específica en la jerarquía conceptual del individuo y son promovidos a la memoria semántica experiencial. Los otros permanecen como elementos de la memoria episódica. Las concepciones alternativas de los alumnos tienen su propia ubicación en la memoria semántica experiencial (de Posada, 1994a), concretamente coincide con la zonal 3 de la figura 2 del modelo propuesto. Más adelante volveremos sobre este punto. Algunos ejemplos de concepciones alternativas son:

- I a idea de que el verano e invierno se producen cuando la Tierra se acerca o se aleja del Sol (Eckrocth, 1993; de Manuel, 1995; Camino, 1995).

- La idea de que las plantas, como seres inferiores, carecen de células (Banet y Ayuso, 1995).

- La concepción continua de la materia para los gases (Novick y Nussbaum, 1981; Furió y Hernández, 1983; Llorens, 1991) y para los sólidos (de Posada, 1993b, 1995).

El individuo, por sí solo, rara vez es consciente de que sus concepciones están en contradicción con lo dictado por las ciencias. Sin embargo, las relaciones incorrectas están dificultando nuevas conexiones significativas con otros conceptos desde un punto de vista acorde con la ciencia (Perkins y Simmons, 1988; Nuthall y Alton-Lee, 1993; Chrobak, 1994).

Como que la memoria semántica academicista es percibida por los alumnos como alejada de lo cotidiano, creemos que la memoria episódica no se encuentra directamente relacionada con aquélta. En cambio, la memoria semántica experiencial puede elevar el nivel de los elementos ubicados en la memoria episódica, integrando en aquélla elementos de ésta, permitiendo la mejora del poder de observación y percepción del mundo que rodea al individuo en los campos relacionados con la memoria semántica experiencial (Villani y Orquiza, 1995; de Posada, en prensa). Los tres tipos de memorias no constituyen grupos separados sino que existe un continuo entre ellos. Los enlaces iónico, covalente y metálico podrían ser símiles útiles para explicar este hecho. Aunque existen sustancias químicas con un tipo de enlace concreto, muchos de los compuestos pueden ser considerados formados por combinaciones entre aquéllos. Algo similar puede ocurrir con estos tres tipos de memorias, en el que las nociones de los individuos pueden ir evolucionando y desplazarse dentro de este sistema.

Hemos podido constatar que las respuestas de los alumnos mejoraban cuando trataban de aplicar algún concepto teórico en un contex to familiar y cotidiano para cllos (de Posada, 1993a; Sanz et al., 1993; Furio et al., 1993; parcialmente en Gómez et al., 1993b). Creemos que experiencias, hechos u objetos próximos a lo cotidiano de los alumnos (que denominamos factores inclusores y no coinciden con los conocimiento previos relevantes descritos por Ausubel et al. [1978]) brindan una mayor oportunidad de producir aprendizajes significativos en los individuos. Esos factores inclusores pueden ser proporcionados por eI medio escolar o extraescolar y deberán estar próximos a la memoria episódica o incluso haber sido registrados por ésta previamente (para más información ver de Posada, 1994b).

\section{MÉTODOS DE ANÁLISIS}

Los modelos exclusivamente semánticos del conocimiento presentan estructuras estáticas. Éstos necesitan contestar cuestiones importantes relacionadas con aspectos dinámicos de la construcción del conocimiento: ¿Cómo los individuos relacionan o conectan experiencias, hechos, conceptos, etc.? ¿Qué tipos de reglas sigue la mente para establecer relaciones? Y de una forma más general: ¿cómo construyen un nuevo concepto científico? La memoria procedimental incluye una amplia varjedad de habilidades, desde cómo montar en bicicleta hasta diseñar una experiencia en la que se puedan controIar algunas variables. Sin desdenar la importancia de las habilidades puramente motoras, en este apartado estudiaremos los tipos de razonamientos o métodos de análisis que solemos utilizar.

Los individuos frecuentemente utilizan en contextos experienciales métodos de análisis superficiales como, por ejemplo, las reglas de accesibilidad, contiguiidad espacial y temporal, semejanza y covariación (Bullock et al., 1982; Kahneman et al., 1982; Carey, 1985; Andersson, 1986; Pozo et al. 1991a,b; Acevedo, 1990). 
Figura 3

Tipos de métodos de análisis utilizados por los individuos.

\begin{abstract}
MÉTODOS DE ANÁLISIS
ACADEMICISTAS

MÉTODOS DE ANÁLISIS EXPERIENCIALES
\end{abstract}

Éstos y otros métodos son los utilizados en el día a día de los individuos y los denominamos métodos de análisis experienciales (Fig. 3). Resulta útil diferenciarlos de otros que serán abordados posteriormente. Estos métodos ayudan a construir conocimientos en los individuos promoviendo elementos inicialmente sueltos e independientes de la memoria episódica hacia la memoria semántica experiencial a través, generalmente, de aprendizajes autónomos. Este hecho lo ilustraré con un ejemplo. Hace algún tiempo, mi hija de 7 años de edad colocó un cubo de hielo en su refresco que estaba a temperatura ambiente. Observó que el hielo se resquebrajó y đe forma espontánea me dijo que el gas del refresco era capaz de romper el hielo. Los aspectos visuales y auditivos son muy accesibles a las personas, en este caso el gas y la ruptura del hielo. Y finalmente existió una contigüiidad espacial y temporal que le llevó a establecer una relación causa-efecto. Cuando estas explicaciones son verbalizadas, el interlocutor (profesor, familiar, compañero, etc.) puede discutirlas y ponerlas a prueba. Sin embargo, los alumnos no exteriorizan continuamente sus ideas, por lo que la recurrencia de experiencias similares sin un control más riguroso de las posibles causas, pueden producir ideas del tipo 3 (Fig. 2) muy asumidas por los individuos.

Iunto con el contenido, escolar y extraescolar, se introducen generalmente de forma no explícita métodos de análisis más elaborados, como proporciones, control de variables, razonamiento hipotético-deductivo, etc. Estos métodos son aprendidos de forma puramente memorística y rutinaria (métodos de análisis academistas, Fig. 3) por gran parte de los alumnos en la enseñanza tradicional, relacionándose esos métodos de análisis exclusivamente con los conceptos específicos impartidos (generalmente elementos de la memoria semántica academicista), ya que no se prueban esos métodos con hechos, experiencias u objetos bien conocidos por los alumnos. Los métodos de análisis academicistas podrían convertirse en experienciales si fueran usados con conceptos pertenecientes a la memoria semántica experiencial de los alumnos de forma reincidente y exitosa. Siegler 1983, 1985, (en Lacasa y Garć́a Madruga, 1992) confirma en parte este hecho exponiendo que sólo mediante la adquisición de un mayor conocimiento son capaces los niños o aduitos de aplicar las reglas más complejas en la resolución de tareas como la del uso de la balanza. Ambos métodos de análisis se necesitan uno al otro para continuar el desarrollo del individuo. Sin el concurso de los métodos de análisis academicistas, no pueden progresar los experienciales $y$, sin una adecuada evolución y maduración de éstos, el aprendizaje se hará

Figura 4

Modelo de estructura cognitiva propuesto para explicar cómo los individuos aprenden ciencia.
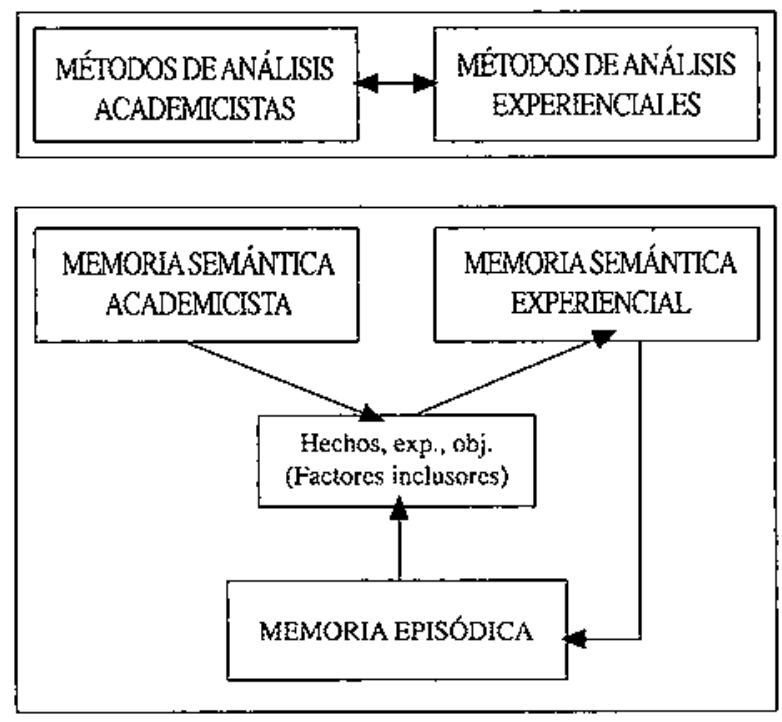

puramente rutinario, con un bajo nivel de retención y aplicabilidad.

En contextos académicos tradicionales, los alumnos tienden a utilizar métodos de análisis academicistas con los elementos de su memoria semántica academicista. En contextos experienciales tienden a utilizar métodos de análisis experienciales sobre elementos de su memoria semántica experiencial. Esto queda esquematizado en la figura 4 y será ilustrado con ejemplos posteriormente en este artículo. Cuando el sujeto no ha asumido un método de análisis academicista adecuado, éste suele hacer uso de uno experiencial para resolver la tarea escolar exigida. De este modo justificamos la fuerte relación encontrada por algunos investigadores entre razonamiento formal y la materia en la que se evalúa éste (Piaget, 1970; Johnson-Laird et al., 1972; Martorano, 1977; Carretero, 1985; Nickerson et al., 1985; García Madruga y Carretero, 1986; Langford, 1989; Stavy, 1990; Niaz, 1991; Acevedo, 1991, entre otros).

Los elementos de la memoria semántica experiencial, tanto del tipo 3 como 4 , han sido conseguidos por el individuo a través de aprendizajes significativos, por tanto se encuentran fuertemente ligados y ampliamente conectados con sus conocimientos previos. Como se recordará, los del tipo 3 son incorrectos y han sido obtenidos en gran medida a partir de la utilización de métodos de análisis experienciales. Es, por tanto, de esperar que los alumnos conf́en ampliamente en esos métodos, en sus observaciones empíricas y en ei producto así obtenido, de forma que no sea fácil modificarlos. Estas predicciones teóricas inferidas a partir del modelo propuesto están de acuerdo con las características recogidas en la bibliografía. En efecto, Driver y otros (1985) 
Figura 5

Diagrama bidimensional orientación de la tarea propuesta / naturaleza de la técnica de extracción de información. Construjodo a partir del diagrama de Driver y Erickson (1983).

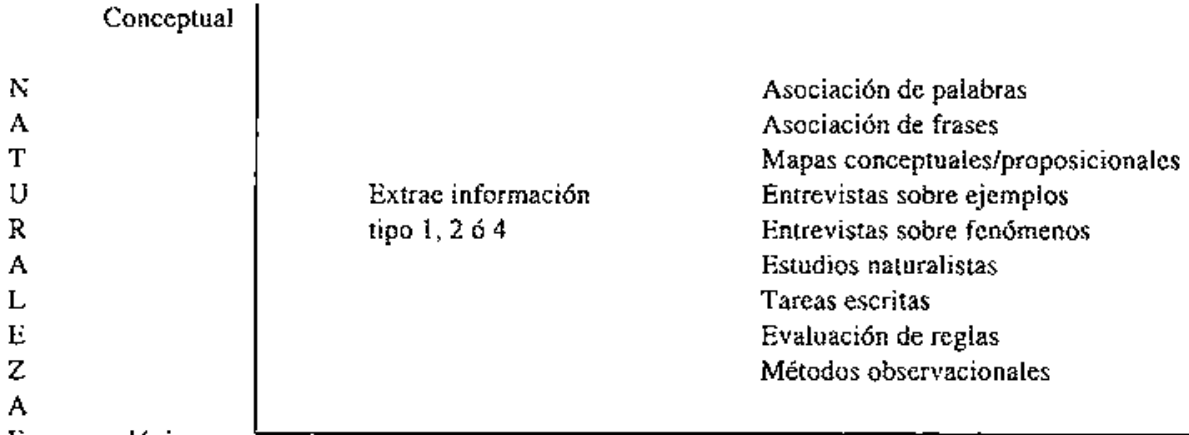

Academicista
ORIENTACION
Fènomenológica
Extrae información tipo 3,4 o procedentes de la memoria episódica

ORIENTACIÓN

No academicista

\begin{abstract}
exponen que las ideas alternativas de los estudiantes son muy tenaces y difíciles de cambiar; Astolfi (1994) argumenta que esas concepciones tienen una red de ideas asociadas; Pozo (1989) expone que tienen una estructura jerarquizada; Driver y otros (1985) manifiestan que, en la producción de esas ideas, el estudiante utiliza secuencias causaies lineales; Gil y Carrascosa (1985, 1990) tas denominan métodos de análisis superficiales; para Solomon (1988), esas ideas son altamente predictivas en el entorno cotidiano del individuo y son inducidas y reforzadas por el lenguaje coloquial. Todos estos resultados experimentales son deducibles del modelo teórico propuesto, por lo que podría constituir un respaldo al mismo.
\end{abstract}

\section{ELEMENTOS DE LA MEMORIA PUESTOS EN JUEGO EN LA CONTESTACIÓN DE TAREAS PLANTEADAS A LOS ALUMNOS}

Se ha comprobado (Piaget, 1972; Driver y Erickson, 1983; Stavy, 1988; Driver, 1988; Song y Black, 1991; de Posada, 1993b, entre otros) que las concepciones científicas de los alumnos son altamente dependientes del contexto en el que deben realizar las tareas que se les solicitan. De acuerdo con Driver y Erickson (1983), la naturaleza de las respuestas depende, en muchos casos, del tipo de técrica utilizada para obtener esa informacion. Estos investigadores propusieron un continuo cntre la estructura conceptual y la fenomenológica. Las técnicas de asociación de palabras y frases se encontrarían a un nivel puramente conceptual, mientras que los métodos observacionales se sitúan a un nivel fundamentalmente fenomenológico. Estos autores lo expresan en los siguientes términos: Sugerimos aqui que las técnicas con estructura conceptual tienden a extraer conocimientos proposicionales en cuanto otras que están estructuradas por una situación o un fenómeno extraen conocimientos en acción» (p. 46, traducción nuestra).
Ese conocimiento en acción supone la aplicación, en el caso práctico propuesto, de concepciones anteriormente asumidas limitada por el método de análisis al alcance del individuo para las nociones implicadas. Determinadas tareas pueden constituir más un problema a resolver que la simple puesta en escena de una concepción concreta, por lo que la inferencia de la concepción en ese caso no resultará fácil, ya que se verá modificada por la acción de numerosas variables. La naturaleza fenomenológica-conceptual de la técnica empleada creemos que puede ser combinada con la orientación academicista/no academicista, percibida por el alumno, en que es colocada la tarea. Representemos en un eje la dimensión "orientación» (academicista/no academicista) y en otro eje la enaturaleza» conceptual/fenomenológica) deI método utilizado para obtener la respuesta (Fig. 5). En el esquema así formado situamos las técnicas expuestas por Driver y Erickson (1983), las colocamos hacia la mediación de la variable orientación, ya que cada una de ellas es susceptible de ser expuesta en un continuo academicista/no academicista. Se extrae un tipo de información u otro dependiendo de la percepción del contexto por parte del individuo, si fue o no impartidoen el currículo, el tiempo transcurrido desde que fue contemplado, etc.; variables que hay que determinar con precision. Hlustraremos este hecho con algunos ejemplos.

\section{Contexto no academicista}

Se han planteado a estudiantes, de diferentes niveles e incluso licenciados (Eckrocth, 1993; de ManueI, 1995; Camino, 1995), preguntas similares a: ¿Por qué hay invierno y verano? El tipo de técnica utilizada fue la discusión de un fenómeno físico mediante pruebas de papel y lápiz o entrevistas; la orientación de las tareas no fue academicista. La respuesta de los estudiantes mayores suele ser que la Tierra se acerca más al Sol en verano 
y se aleja en invierno. El indiviđuo utiliza la noción de sistema solar que se encuentra relacionada con hechos y experiencias bien conocidos y asumidos en estudiantes de esta edad; por tanto, se encuentra en su memoria semántica experiencial. Para llegar a esta conclasión, el sujeto hace uso de un método de análisis causal simple: la semejanza. Éste sabe que cuando nos acercamos a un foco de calor (fogata, cerilla, calefactor, etc.) sentimos aumentar la temperatura y cuando nos alejamos ocurre lo contrario.

Veamos qué ocurre cuando se plantea la misma cuestión a un alumno mucho más joven ( 7 años y 6 meses de edad).

\begin{abstract}
A. Porque si hubiera sólo invierno no pasariamos un poco de calor, tendriamos que ir siempre abrigados. Y si sólo hubiera verano nosotros no íbamos a tener un poco de frío. (Silencio). Algunas veces pasa frío en verano sólo que son muy pocas veces.
\end{abstract}

\section{E. El sol tiene algo que ver con que haya invierno y verano?}

A. Porque en invierno las nubes se tapan, tapan el Sol y se queda un poco frío. En invierno muchas veces tapa el Sol.

\section{E. ¿Has oido decir que la Tierra gira alrededor del Sol?}

A. Todo el mundo lo sabe. Hasta los alumnos de preescolar lo saben.

E. ¿Tendrá algo que ver esa vuelta de la Tierra alrededor del Sol con lo que estamos hablando?

\section{A. Sí. Pero no lo sé.}

En la primera respuesta el alumno expone elementos episódicos fruto de un análisis típicamente egocentrista que ya fue caracterizado por Piaget. El alumno percibe al final de su respuesta que ésta no es demasiado coherente y trata esos casos como excepciones. El entrevistador introduce un elemento externo y el alumno desecha fácilmente su visión egocentrista. En la segunda respuesta el alumno utiliza una noción más elaborada construida en el cotidiano: los días de invierno suelen estar más nublados que los de verano. El método de análisis utilizado en este caso es la covariación simple por la que dos hechos que suceden generalmente juntos como días nublados y días fríos de invierno $y$, al contrario, son tratados como causa-efecto. Finalmente, el entrevistador introduce la idea de sistema solar que parece conocida por el alumno. Sin embargo, no es atifizada en sus explicaciones. Este conocimiento no es aún operativo para el alumno; no le sirve para deducir o inferir razonamientos. Incluso siendo el mismo que el de los adultos, no tiene cualitativamente sus mismos atributos. No ha sido incorporado substantivamente a lo que ya conoce, se encuentra, por tanto, aislado en su memoria ${ }^{4}$. Se trata de un conocimiento que se sitúa en la memoria semántica academicista del individuo de este nivel de escolarización. Es generalmente de la utilización de concepcio- nes ubicadas en la memoria semántica experiencial (tipos 3 y 4, Fig. 2) a través de métodos de análisis experienciales como el individuo da una respuesta que considera plausible en el contex to no academicista considerado (Fig. 4). En otras palabras, se pueden obtener respuestas que supongan desde ideas, recuerdos, experiencias, etc. sueltas sin conexión entre sí, hasta concepciones altamente significativas para el individuo.

\section{Contexto academicista}

Veamos ahora el caso contrario, una tarea de lápiz y papel planteada con orientación estrictamente académica: ¿Qué ocurrirá con las siguientes sustancias cuando intentemos disolverlas en agua? $\mathrm{Na}_{2} \mathrm{~S} ; \mathrm{Ca} ; \mathrm{I} \mathrm{Cl} ; \mathrm{Ni}$. Esta cuestión fue planteada a alumnos de $2^{\circ}$ de BUP (aproximadamente de 15 años de edad) antes de comenzar el tema đel enlace químico (de Posada, 1993a). Los alumnos percibieron rápidamente que no existía relación entre las sustancias propuestas y las conocidas por él en el día a día, reforzando la orientación escolar de la tarea pianteada. Aproximadamente el $40 \%$ de esos alumnos no respondi 6 , aduciendo en muchos casos no conocer las sustancias propuestas. EI $30 \%$ aproximadamente

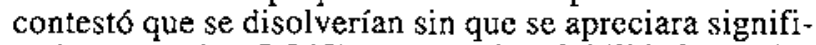
cativamente $(p=0,048)$ patrones de solubilidad entre las sustancia sino que probablemente fueran respuestas aleatorias. El $3 \%$ proponía reacciones químicas para la disolución y el $20 \%$ aproximadamente daba multifad de esquemas diferentes, entre ellos la fusión del soluto en agua o como lo solían denominar «el sólido se derrite».

Esta tarea no es especialmente difícil si es planteada inmediatamente después de introducir el tema, ya que se hacen multitud de ejercicios parecidos a éste. Para la correcta solución, el alumno ha de localizar los elementos en la tabla periódica, utilizar reglas que relacionen la posición en la tabla periódica y el tipo de sustancia, hacer uso de reglas que relacionen tipo de enlace con la solubilidad en diferentes disolventes. Además, deberá construir proposiciones lógicas con la información secuencialmente obtenida y extraer finalmente conclusiones. En todo este proceso pueden producirse errores, como en el caso siguiente: Para que una sustancia pueda disolverse tiene que formar enlace iónico. El $\mathrm{Na}_{2} \mathrm{~S}$ es un enlace covalente al igual que el $\mathrm{Cl}$, mientras que el $\mathrm{Ca}$ y el Ni forman enlace iónico; por lo tanto, ninguna sustancia se disolverá en agua (Alumno de química de COU, 17 años).

Para la mayoria de los estudiantes del nivel considerado, los conceptos utilizados se encuentran ubicados en su memoria semántica academicista (tipo I ó 2, Fig. 2); tan sólo los alumnos capaces de realizar aprendizaje significativo ${ }^{5}$ darán contestaciones del tipo 4 incluso bastante tiempo después de haber sido contemplados esos conceptos en el currículo.

Fue planteada la cuestión aproximadamente un mes después de haber dado el tema de enlace químico (de Posada, 1993a). Tras largos períodos de tiempo son 
olvidados tos elementos semánticos 1 y 2 y los métodos de análisis academicistas. Con esta cuestión, el alumno se enfrenta a sus propios recursos y concepciones interiorizadas. Analicemos la respuesta dada por un alumno de $3^{\circ}$ de BUP de 16 años: El calcio no se disuelve porque es un metal que está sólido a la temperatura que el agua está líquida. I Cl se disuelve porque tiene enlace covalente y su punto de fusión y ebullición es bajo como el agua. El niquel no se disuelve porque es otro metal que está sólido a la temperatura en que el agua está líquida. Resulta complejo entender las razones aducidas, por un Lado utiliza adecuadamente el tipo de enlace y la previsión de los puntos de fusión y ebullición, pero por otro, se relacionan éstos con la solubilidad en agua. El criterio utilizado fue que las sustancias con puntos de fusión parecidos al agua conseguirán disolverse en ella y las que lo tengan muy diferentes no podrán, idea común en alumnos más jóvenes. Las reglas de solubilidad no fueron interiorizadas por este estudiante que vuelve a utilizar la que ya poseía, según ésta el soluto «se derrite» o funde en agua al disolverse.

Cuando los individuos tienen que realizar una opción en su día a día, no suelen utilizar un procedimiento de análisis excesivamente elaborado que lleve tiempo aplicar, ya que la mayor parte de las personas, adultos incluidos, siguen confiando en otras reglas más simples que, aunque potencialmente incorrectas, son habitualmente eficaces (Tversky y Kahneman, 1974; Pozo et al., 1991 a); sólo cuando perciben la ineficacia o contradicción es cuando hacen uso de otros métodos de análisis más elaborados, como pudimos observar en la entrevista con el alumno de siete años sobre las causas que ocasionan los inviernos y veranos. Con cierto esfuerzo, los estudiantes consiguen retener de forma rutinaria información que es almacenada como memoria semántica academista y métodos de análisis academistas, si éstos no consiguen integrarse en la memoria semántica experiencial y métodos de análisis experienciales, se corre el riesgo de olvidar. Si esto ocurre y esa información es requerida, Ias respuestas de Ios alumnos suelen ser concepciones alternativas, las cuales no son más que métodos de análisis experienciales actuando sobre elementos de la memoria semántica experiencial. Como en el caso del alumno de $3^{\circ}$ de BUP, que pudo integrar elementos científicos correctos pero las razones que producen la solubilidad en agua permanecieron esencialmente inalteradas.

\section{COMENTARIOS FINALES}

Entendemos que cada una de las principales teorías constructivistas describe perspectivas diferentes de la panorámica del aprendizaje humano bastante válidas en sus respectivos ámbitos de aplicación. Creemos que es posible armonizar numerosos puntos de esas teorías dentro de un nuevo marco, aunque somos conscientes de que subyacen concepciones filosóficas muy diferentes en ellas (Nussbaum, 1989). Sólo tendremos una noción de conjunto cuando integremos y limemos las asperezas entre las distintas perspectivas. Esta tarea no es simplemente la yuxtaposición sin más de las teorías existentes, sino la aglutinación dentro de un marco apropiado para tal fin.

La diferenciación de la memoria en tres niveles: semántica academicista, semántica experiencial y episódica, junto con la división de los métodos de análisis utilizados por los individuos en experienciales y academicista, puede constituir el marco apropiado para la confección de una teorfa aglutinadora sobre el aprendizaje de conceptos científicos. Con este nuevo modelo podemos dar explicación a hechos experimentales procedentes del campo que se ha venido en denominar ideas alternativas de los alumnos y que no eran suficientemente justificados por otras teorías constructivistas.

Algunas características de las ideas de los alumnos descritas por Driver y otros (I985) han sido reinterpretadas a lo largo de este artículo:

- La percepción cotidiana es importante en la formación de recuerdos, experiencias, hechos, etc. inicialmente independientes entre sí, ubicados en la memoria episódica del individuo.

- Es a través de la atención prestada por el individuo a algunos elementos de la memoria episódica, generalmente llamativos, mediante el uso de métodos de análisis experienciales (con frecuencia razonamientos causales simples) cómo los sujetos van construyendoelementos de la memoria semántica experiencial más elaborados y asumidos.

- Estas ideas inicialmente indiferenciadas así formadas van ganando precisión gracias a la acción del aprendizaje significativo que se produce en los entornos tanto escolares como extraescolares. Para que se produzca aprendizaje significativo será necesario que: se activen ideas inclusoras, los métodos de análisis requeridos se encuentren al nivel alcanzado por el alumno, haya suficiente motivación, tenga autoestima, actitud positiva, percepción favorable del contexto, refuerzos periódicos de las ideas correctas sobre las equivocadas, etc. (de Posada, en prensa).

- El pensamiento de los alumnos depende del contexto en que se active. Desde nuestro marco teórico se han tipificado los elementos de la memoria y métodos de análisis utilizados por los individuos para dar respuesta a tareas planteađas con orientación academicista y no academicista.

El modelo propuesto constituye un marco teórico de aprendizaje de conceptos científicos que no implica necesariamente un modelo de enseñanza. La transposición no puede ser directa; sin embargo, podrian extraerse algunas implicaciones valiosas. Animamos desde aqui a los lectores curiosos a que apliquen el modelo a sus datos experimentales para comprobar la utilidad. También alentamos a los investigadores teóricos a que pongan a prueba la validez de los presupuestos que la sustenta. 
A nuestro juicio, son resueltas algunas deficiencias pero se suscitan nuevos problemas, que el lector atento habrá detectado, y que precisarán nuevas soluciones. A pesar de los múltiples defectos que pueda tener el uso de símiles, me atrevería a decir que, en la didáctica de las ciencias nos encontramos explorando «la estructura terciaria y cuaternaria de las proteínas»; es decir, no descendemos a lo que ocurre en la mente del individuo en el transcurso de segundos o fracciones -memoria a corto plazo y memoria de trabajo-, lo cual equivaldría al «estudio de la estructura primaria y secundaria polipetídica». Dicho en otras palabras, tenemos multitud de conocimientos correctos pero sectarios y no se realiza una integración satisfactoria de esa información, por lo que nuestro conocimiento es aún muy limitado.

La principal crítica que hemos recibido hasta ahora es la de que el modelo ha sido confeccionado, aparentemente ad hoc, a partir de las principales teorías constructivistas para explicar hechos experimentales relacionados con las concepciones alternativas de los alumnos. Aunque fue efectivamente éste nuestro interés inicial, creemos que es necesaria y posible la integración de las diferentes corrientes dentro de un marco más general. Ese marco podría ser éste u otro que se establezca en su día, pero sin duda merece la pena invertir esfuerzos en conseguirlo. Animamos desde aquí a los diferentes investigadores en este campo a trabajar en ello.

\section{REFERENCIAS BIBLIOGRÁFICAS}

ACEVEDO, J. A. (1990). Razonamiento causal en una tarea de contexto natural. Un estudio evolutivo con estudiantes de bachillerato. Investigación en la Escuela, 10, pp. 61-70.

ACEVEDO, J. A. (1991). Patrones de razonamiento proporcional en la resolución de tareas de ciencias. Suma, 8, pp. 41-47.

ADEY, P.S. (1987). A response to «Towards a Lakatosian analysis of Piagetian and alternative conceptions research programs». Science Education, 71, pp. 5-7.

ALEXANDER,P.A., SCHALI.ERT, D.L. y HARE, V.C.(1991) Coming to terms: How researchers in learning and literacy talk about knowledge. Review of Educational research, 61, pp. 315-343.

ANDERSON, B.F. (1975). Cognitive Psychology: The study of knowing, learning and thinking. Nueva York: Academic Press.

ANDERSON, J.R. (1980). Cognitive psychology and its implications. San Francisco: Freeman.

ANDERSON, I.R. (1983). The architecture of cognition. Cambridge, Ma.: Harvard University Press.

ANDERSSON, B. (1986). The experiential gestalt of causation: a common core to pupils' preconceptions in science. European Journal of Science Education, 8(2), pp. 155-171.

\section{AGRADECIMIENTOS}

Quisiera dar las gracias a Carmen P. de Aranega, Nicolás Marín, José María Oliva y José Antonio Acevedo por sus valiosos comentarios al primer borrador de este artículo.

\section{NOTAS}

- Marín (1994b) cree que no es posible una relación completa entre los términos de Piaget (1971) figurativo y operativo y los términos declarativo y procedural de Ryle (1949).

2 El conocimiento jerarquizado del individuo ha sido expuesto, entre otros, por Vygotsky (1962), Ausubel y otros (1978), Piaget (1975), las teorías del procesamiento de la información y el movimiento de las concepciones alternativas.

3 El papel preponderante de la propia experienciaen el aprendizaje viene siendo resaltado por la pedagogía desde hace mucho tiempo (Pestalozzi, Freinet). EI concepto de aprendizaje significativo tiene una gran tradición; las primeras ideas proceden de los trabajos de Wertheimer (1945), y más tarde fue profundizado por Ausubei y otros (1978) y Novak y Gowin (1984).

4 Nussbaum (1985) comprobó que el $45 \%$ de los estudiantes israelíes de 8 años de edad consideran la Tierra plana. Casi el $40 \%$ cree que es una bola con el hemisferio sur de roca y tierra, y la parte norte de aire.

5 Con un enfoque en profundidad de la materia, de acuerdo con la terminología de Marton (Coll, 1991b).

ASTOLFI, J.P. (1994). El trabajo didáctico de los obstáculos, en el corazón de los aprendizajes científicos. Enseñanza de las Ciencias, 12(2), pp. 206-216.

AUSUBEL, D.P.; NOVAK, J.D. y HANESIAN, H. (1978). Educational Psychology. 2a. ed. (Nueva York: Holt, Rinehart y Winston.). Trad, cast. de Sandoval, M. Psicologia educativa: Un punto de vista cognoscitivo. México, 1983: Trillas.

BADDELEY, A. (1994). Las memorias humanas. Mundo Cientifico, $150(14)$, pp. 802-807.

BANET, E. y AYUSO, E. Introducción a la genética en la ensenanza secundaria y bachillerato: I. Contenidos de enseñanza y conocimientos de los alumnos. Enseñanza de las Ciencias, I3(2), pp. 137-154.

BEREISTER, C. (1990). Aspects of an educational learning theory. Review of Educational Research, 60, pp. 603-624.

BULLOCK, M., GELMAN, R. y BAILI,ARGEON, R. (1982). The development of causal reasoning, en Friedman, W.J. The developmental psychology of time. Londres: Academic Press.

CALFEE, R. (1981). Cognitive psychology and educational practice, en Berliner, D.C. (ed.), Review on research in education, 9, pp. 3-73. Washington, D.C.: American Educational Research Association. 
CAMINO, N. (1995). Ideas previas y cambio conceptual en astronomía. Un estudio con maestros de primaria sobre el día y la noche, las estaciones y las fases de la Luna. Enseñanza de las Ciencias, 13(1), pp. 81-96.

CARDEBAT, D., DÉMONET, I.F, y PUEL, M. (1994). Los trastornos del sentido de las palabras. Mundo científico, 150) (14), pp. 864-869.

CAREY,S.(1985). Conceptual change in childhood Cambridge, MA.: MI'T Press.

CARRETERO, M. (1985). El desarrollo cognitivo en la adolescencia y la juventud: las operaciones formales, en Carretero, M., Palacios, J. y Marchesis, A. Psicologia evolutiva 3. Adolescencia, madurez y senectud. Madrid: Alianza.

CHROBAK, R. (1994). Introducing meaningful learning tools in introductory physics courses. Actas de la Conferencia Internacional Science and Mathematics Education for the 21st Century: Towards innovatory approaches, pp. 321 337. Chile: Universidad de Concepción.

COLL SALVADOR, C. (1991a). Concepción constructivista y planteamiento curricular, Cuadernos de Pedagogía, 188, pp. 8-11.

COLI, SALVADOR, C. (1991b). Aprendizaje escolar y construcción del conocimiento (2a. ed.). Barcelona: Paidós Educador.

COLLINS, A.M. y QUILLIAN, M.R. (1969). Retrievat time from semantic memory. Journal of Verbal Learning and Verbal Behavior, 8, pp. 240-247.

CORNO, L. (1981). Cognitive organizing in classrooms. Curriculum Inquiry, $1 \mathrm{I}$, pp. 359-377.

CORRAL IÑIGO, A. (1986). El funcionamiento cognitivo del adulto. Madrid: ICE de la UNED.

DRIVER, R. (1988). Un enfoque constructivista para el desarrollo del curcucho en ciencias. Enseñanza de las Ciencias, $6(2)$, pp. $109-120$.

DRIVER, R. y ERICKSON, G. (1983). Theories-in-action: Some theoretical and empirical issues in the study of students ${ }^{*}$ conceptual fremeworks in Science. Studies in Science Education, 10, pp. 37-60.

DRIVER, R., GUESNE, E. y TIBERGHIEN, A (1985). Children's ideas in Science. Glasgow: Milton Keynes, Open University Press.

ECKROTH, C.A. (1993). The Earth's seasons in 3-D. Part 1. The physics Teacher, 31 (oct), pp. 419-420.

FURIÓ, C. y HERNÁNDEZ, J. (1983). Ideas sobre los gases en alumnos de 10 a 15 años. Enseñanza de las Ciencias, 1, pp. 83-91.

FURIÓ, C., AZCONA, R., GUISASOLA, G. y MUJIKA, E. (1993). Concepciones de los estudiantes sobre una magnitud «olvidada» en la enseñanza de la química: la cantidad de sustancia. Enseñanza de las Ciencias, 11(2), pp. 107-114.

GAGNF, R.M., BRIGGS,I.J.y WAGER,W.W.(1988). Principles of Instructional Design. (3a.ed.). Nueva York: Holt, Rinehart y Winston.

GARCIA MADRUGA, J.A. y CARRETERO, M. (1986). Estrategias en el razonamiento humano: tareas logicas y probabilísticas, en Peraita, H. Psicologia congestiva y ciencia congestiva. Madrid: UNED.

GIL, D. y CARRASCOSA, J. (1985). Science Learning as a conceptual and methodological change. European Journal of Science Education, 7(3), pp. 231-236.
GIL, D. y CARRASCOSA, J. (1990). What to do about Science «Misconceptions». Science Education, 74(5), pp. 531-540.

GILBERT, J.K. y SWIFT, D.J. (1985). Towaros a Lakatosian analysis of the Piagetian and alternative conceptions research programs. Science Education, 69, pp. 681-696.

GIORDAN, A. y de VECCHI, G. (1988). Los origenes del saber. De las concepciones personales a los conceptos cientificos. Sevilla: Díada.

GÓMEZ, M.A., POZO, J.I.y SANZ, A. (1993a). Lacomprensión de la conservación de la materia en problemas de disoluciones. Enseñanza de las Ciencias. IV Congreso Internacional sobre investigación en la Didáctica de las Ciencias y de las Matemáticas. pp. 237-238.

GOMEZ, S., LATORRE, A. y SANJOSÉ, V. (1993b). EI modelo de Ausubel en la didáctica de la física: Una aproximacion experimental al proceso de E/A de contenidos que presentan constructor pocoelaborados por los aprendices. Enseñanza de las Ciencias, 11(3), pp. 235-246.

JOHNSON-LAIRD, P.N., LEGRFNZI, P. y SONINO LEGRENZI, M. (1972). Reasoning and a sense of reality. British Journal of Psychology, 63, pp. 395-400.

JOHNSON-LAIRD, P.N. (1983). Mental models: Towards a cognitive science of language, inference and conscionsness. Cambridge, R.U.: Cambridge University Press.

KAHNEMAN, D., SLOVIC, P. y TVERSKY, A. (1982). Judgment under uncertainy: heuristic and biases. Cambridge: Cambridge University Press.

LACASA, P. y GARCÍA MADRUGA, J.A. (1987). Algunos modelos teóricos recientes en el estudio del desarrollo cognitivo, en Carretero, M., García Madruga, J.A., Herici, C., Lacasa, P., Peraita, H., Santa Cruz., J., y Sierra Vázquez, V. Psicología congestiva y ciencia congestiva. Madrid: UNED.

LACASA, P. y GARCÍA MADRUGA, J.A. (1992). El desarrollo de la memoria: Estrategias y conocimiento, en: García Madruga, J.A., y Lacasa, P., Psicología evolutiva. Desamollo cognitivo y social. 1a. reimpresión. Madrid: UNED.

L.ANGFORD,P.(1989). El desarrollodel pensamiento conceptual en la escuela primaria. Barcelona: Paidós.

LAWSON, A.E. (1982). The reality of General Cognitive Operations. Science Education. 66(2), pp. 229-241.

LAWSON, A.E. (1989). Author's response. Journal of Research in Science Teaching, 26, pp. 825-826.

LEVINE, D.S. y PRUEITT, P.S. (1989). Modeling some effects of frontal lobe damage: Novelty and perseveration. Neural Networks, 2, pp. 93-116

IURIA (1973). The working brain: An introduction for neuropsychology. Nueva York: Basic Books.

I.LORENS, J.A. (1991. Comenzando a aprender química. Ideas para el diseño curricular. Madrid: Visor.

MANUEL, J. DE. (1995). ¿Por qué hay veranos e inviemos? Representaciones de los estudiantes (12-18) y de futuros maestros sobre algunos aspectos del modelo Sol-Tierra. Enseñanza de las Ciencias, 13(2), pp. 227-236.

MARÍN, N. y BENAROCH, A. (1994a). A comparative study of Piagetian and constructivist work on conceptions in science. International Jokmal of Science Education, $16(1)$, pp. $1-15$.

MARÍN, N, (1994b). Comunicación personat. 
MARÍN, N. y JIMÉNEZ, E. (1992). Problemas meteorologicos en el tratamiento de las concepciones de los alumnos en el contex to de la filosofía e historia de la ciencia. Enseñanza de las Ciencias, 10(3), pp. 335-339.

MARTORANO, S.C. (1977). A developmental analysis of performance on Piaget's formal operational tasks, Developmental Psychology, 13, pp. 666-672.

NIAZ, M. (I991). Correlates of formal operational reasoning: a neo-piagetian analysis, Journal of Research in Science Teaching, $28(1)$, pp. 19-40.

NIA7, M. (1993). "Progressive problemshifts» Between Different Research Programs in Science Education: A Lakatosian Perspective. Journal of Research in Science Teaching, $30(7)$, pp. 757-765

NICKERSON, R.S., PERKINS, D.N. y SMITH, E.E. (1985). The teaching of the thinking. Nueva Yersey: Lawrence Eribaum Associates Publishers. Trad cast. de Romano, L. y Ginard, C., Enseñar a pensar. Aspectos de la aptitud intelectual. Barcelona (1987): Paidos. MEC.

NOVAK, J.D. (1988). Learning Science and the Science of Learning. Studies in Science Education, 15, pp. 77-101.

NOVAK, J.D. y GOWIN, D.B. (1984). Learning how to learn. Nueva York: Cambridge University Press.

NOVICK, S. y NUSSBAUM, J. (1981). Pupils'understanding of the particulate nature of matter: A cross-age study. Science Education, 65(2), pp. 187-196.

NUSSBAUM, K. (1985). The Earth as a Cosmic Body. Chapter 9 , en Driver, R., Guesne, E., y Tiberghien, A., Children's ideas in Science. Glasgow: Milton Keynes, Open University Press.

NUSSBAUM, J, (1989). Classroom conceptual change: philosophical perspectives. International Joumal of Science Feducation, li (special issues), pp. 530-540.

NUTHALL, G. y ALTON-LEE, A (1993). Predicting Learning From Students Experiece of Teaching: A Theory of Student Knowledge Construction in Classroom. American Educational Reseurch Journal, 30(4), pp. 799-840.

OSBORNE, R. y WITTROCK, M. (1983). Learning science A generative process. Science Education, 67(4), pp. 489-508.

OSBORNE, R. y WITTROCK, M. (1995). The generative learning model and its implications for science education. Studies in Science Education, 12, pp. 59-87.

PÉRĚZ GÓMFZ, A.I. (1988). Memoria del curso de doctorado «Estrategias, técnicas e instrumentos de la investigación etnográfica en la escuela». Material fotocopiado. Universidad de Málaga

PERKINS, D.N. y SIMMONS, R. (1988). Patterns of misunderstanding: An integrative model for science, maths and programming. Review of Educational Research, 58, pp. 30.3-326.

PIAGET, J. (1970). L, evolution intellectuelle entrel' adolescence et l'age aduite. Rapport sur le III Congrés International FONEME sur la formation humaine a l’age adulte. Mílán.

PIAGET, J. (1971). (Genetic epistemology. Nueva York: Norton.

PIAGET, J. (1972). Human development. 15(1).

PIAGET, I. (1974). Understanding causality. Nueva York: W.W. Norton.
PIAGFT, J. (1975). L'équilibration des structures cognitives. Probleme central du developpement. París: PUF.

PORI.ÁN, R. (1993). La didáctica de las ciencias, Cuadernos de Pedagogia, 210 , pp. 68-71.

POSADA, J.M. DE (1993a). Estudio de los constructos de los alumnos y análisis secuencial de libros de texto en los niveles de BUP y COU con zelación a la estructura de la materia y entace químico. Tesis doctoral no publicada. Universidad de Mátaga. Breve resumen en Enseñanza de las Ciencias, 11(3), pp. 351-353.

POSADA, J.M. DE (1993b). Concepciones de los alumnos do 15-18 años sobre la estructura interna de la materia en el estado sólido. Enseñanza de las Ciencias, 11 (1), pp. 12-19.

POSADA, J.M. DE (1994a). Towards an aglutinative constructivist theory of the pupil conception in Science. Actas de la Conterencia Internacional Science and Mathematics Education for the 21 st Century: Towards innovatory approaches, $\mathrm{pp}$. 505-512. Chile: Universidad de Concepción.

POSADA, J.M. DE(1994b). Un punto noresuelto en la teoría de Ausubel: la relación entre clementos experienciales y aprendizaje significativo. Actas de la Conferencia Internacional Science and Mathematics Education for the 21st Century: Towards innovatory approaches, pp. 513-516. Chile Universidad de Concepción.

POSADA, J.M. DE (1995). Contenidos, actividades o estrategias de enseñanza de la química en la formación científica de ciudadanos. Noticias Pan-Americanas en educación química 7(1), pp. 2-3.

POSADA, J.M. DE (En prensa). Un modelo psicopedagógico aglutinador para el aprendizaje de la física. Revista de Enseñanza de la Física.

POSNER, G.J., STRIKE, K.A., HEWSON, P.W. y GERTZOG W.A. (1982). Accommodation of a Scientific Conception Toward a Theory of Conceptual Change. Science Education, $66(2)$, pp. 211-227.

POZO, J. I. (1989). Teorias cognitivas del aprendizaje. Madrid: Morata, SA.

POZO, J.I., GÓMEZ, M.A., LIMÓN, M. y SANZ, A. (1991a) Procesos cognitivos en la comprensión de la ciencia: las ideas de los adolescentes sobre la química. Madrid: CIDE. Centro de publicaciones del MEC.

POZO, J.I., SANZ, A., GÓMEZ, M.A. y LIMÓN, M. (1991b) Las ideas de los alumnos sobre la ciencia: una interpretación desde la psicología congestiva. Enseñanza de las Ciencias, 9(1), pp. 83-94.

RIDLEY,D.R. y NOVAK, J.D. (1983). Sex-related differences in high schools science and mathematic enrollments: Do they give males a critical head start toward science -and math-related careers? The Alberta Journal of Educational Research, 24(4), pp. 308-318.

RIPS, L.J., SHOBEN, E.J. y SMITH, E.E. (1973). Semantic distance and the verification of semantic relations. Journal of Verbal Learning and Verbal Behavior, 12, pp. 1-20.

RYLE, G. (1949). The concept of mind. Nueva York: Penguin Books

SANZ, A., POZO, J.I., PẾRFZ ECHEVFRRÍA, M.P. y GÓMEZ, M.A. (1993). Influencia de la instrucción y el contenido en el razonamiento proporcional. Enseñanza de las Ciencias. IV Congreso Internacional sobre investigación en la Didáctica de las Ciencias y de las Matemáticas, $\mathrm{pp}$. 349-350. 
SEBASTIÁN, M.V. (1983). Lecturas de psicologiadela memoria (compilación). Madrid: Alianza.

SOLOMON, J. (1988). Una perspectiva social de los esquemas conceptuales. Investigación en la Escuela, 5, pp. 17-20.

SONG, J y BLACK, P.J. (1991). The effects of task contexts on pupils' performance in science process skills. International Joumal of Science Education, 13(1), pp. 49-58.

STAVY, R. (1988). Children's conception of gas. International Journal of Science Education, 10(5), pp. 553-560.

STAVY, R. (1990). Pupils' problems in understanding conservation of matter. International Journal of Science Education, 12(3), pp. 501-512.

STREIBHL, M.J. (1989). Diseño instructivo y aprendizaje situado: ¿Hs posible un maridaje? Revista de Educación, 289 , pp. 215-234.

TOBIN, K. y FRASER, B.J. (1989). Barriers to higher-level cognitive learning in high school sciencc. Science Education, 73(6), pp. 659-682.

TULVING, E. (1972). Fpisodic and semantic memory, en Tulving, E.W. y Donalson, W. (eds.). Organization of Memory. Nueva York: Academic Press.
TULVING, E. y THOMSON, D.M. (1973). Fncoding specificity and retrieval processes in cpisodic memory. Psychologicul Review, 80, pp. 352-373.

TVERSKY, A. y KAHNEMAN, D. (1974). Judgements under uncertainty: Henristic and biases. Science, 185. Trad. cast. de Pozo, J.I., en Lecturas de Psicologia del pensamiento. Madrid, 1984: Alianza.

VILI,ANI, A. y ORQUIZA, I.. (1995). Evolución de Ias representaciones mentales sobre colisiones. Documento interno. Brasil: Universidad de San Pablo.

VUYK, R. (1985). Panorámica y crítica de la epistemología genética de Piaget, 1965-1980. Madrid: Alianza Universitaria.

VYGOTSKI, L.S. (1962). Thought and language. Cambridge: MI'. Press. Trad. port de Camargo, J.I.,. Pensamento e linguagem. San Pablo (Brasil): Martins Fontes (1991).

WERTHEIMER, M. (1945). Productive thinking. Chicago: The University of Chicago Press.

WHITE, R. T. (1988). Leaming Science. Oxford: Basil Blackwel] Ltd.

[Artículo recibido en febrero de 1994 y aceptado en julio de 1996.] 